\title{
Cardiovascular Disease in Chronic Kidney Disease: Review Article Abdulrhman Al Abdulqader
}

Department of Internal Medicine, King Faisal University, Al-Ahsa, Saudi Arabia

Corresponding author: Abdulrhman Al Abdulqader, Mobile: +966555917740, E-Mail: alabdulqader@kfu.edu.sa

\begin{abstract}
Background: Since the first description of the association between chronic kidney disease and heart disease, many epidemiological studies have confirmed and extended this finding. As chronic kidney disease progresses, kidneyspecific risk factors for cardiovascular events and disease come into play. As a result, the risk for cardiovascular disease is notably increased in individuals with chronic kidney disease.

Objective: To study the relationship between importance of CVD in determining of CKD patients.

Conclusion: Cardiovascular disease is frequently underdiagnosed and undertreated in patients with chronic kidney disease. This group of patients should, therefore, be acknowledged as having high cardiovascular risk that needs particular medical attention at an individual level.
\end{abstract}

Keywords: Cardiovascular Disease, Chronic Kidney Disease.

\section{INTRODUCTION}

Chronic kidney disease (CKD) is an independent risk factor for the development of coronary artery disease, and for more severe coronary heart disease (CHD). CKD is also associated with adverse outcomes in those with existing cardiovascular disease (1). This includes increased mortality after an acute coronary syndrome, after percutaneous coronary intervention (PCI) with or without stenting, and after coronary artery bypass. In addition, patients with CKD are more likely to present with atypical symptoms, which may delay diagnosis and adversely affect outcomes ${ }^{(2)}$.

Fink et al. ${ }^{(3)}$, reported that cardiovascular disease (CVD), as it is well known, was the leading cause of death in chronic kidney disease (CKD). They also stated that risk factors associated with CVD fully exists within CKD patients. These include accelerated atherosclerosis, left ventricular hypertrophy (LVH) and dilatation, systolic dysfunction, and high left ventricular filling pressure. They deduced that cardiac disorders and renal diseases affect each other through direct and indirect complex mechanisms. According to Keith et al. ${ }^{(4)}$, the high mortality rates occurring among patients having CKD stage 5 and stage $5 \mathrm{D}$, are mainly due to cardiovascular disorders co-existing with chronic kidney disease and end-stage renal stage.

\section{Proteinuria/Albuminuria and CVD:}

The presence of a protein quantity in urine exceeding normal range is called (proteinuria), and when this protein consists mainly of albumin, it is called (albuminuria). We can detect proteinuria by urine dipstick testing in a semi-quantitative way, by measuring protein/creatinine ratio (UPCR) or urinary albumin/creatinine ratio (UACR), or by quantifying protein in collected 24 hours urine using laboratory methods. In diabetic nephropathy patients, we use micro-albuminuria in the range of $30-300 \mathrm{mg} / \mathrm{day}$ (albumin in urine) and overt albuminuria as > 300 $\mathrm{mg} /$ day (albumin in urine), while on assessing nondiabetic CKD patients, we use a range of proteinuria > $300 \mathrm{mg} /$ day or urinary protein/creatinine ratio > 200 $\mathrm{mg}$. Proteinuria is either considered to be due to renal damage or a systemic endothelial dysfunction marker. Proteinuria accompanied cardiovascular disease and mortality, in general population, even at values regarded as normal. The presence of reduced eGFR, associated with micro- or macro-albuminuria, were shown within several studies to be independent predictors of mortality, either in diabetics, or in nondiabetics as reported ${ }^{(5,6)}$.

\section{GFR and CVD:}

GFR decline was proved to be an independent risk factor for cardiovascular morbidity and mortality, wither proteinuria is present or not. Kottgen et al. ${ }^{(7)}$, in their study including more than one million subjects above 18 years, found that eGFR decline affected cardiac and vascular system state of patients leading to cardiovascular events such as CAD, heart failure, stroke, peripheral vascular disease and death, as reported within ARIC study (Atherosclerosis Risk in Community Study).

\section{Cardiovascular Injury in CKD:}

Pathogenetic mechanisms leading to cardiovascular damage in renal disease were reported that more than a dozen of pathways were recognized including Renin-Angiotensin-Aldosterone System (RAAS) hyperactivity, osmotic sodium retention volume overload, endothelial dysfunction, dyslipidemia, coagulopathy, inflammation and anemia. The following pathogenetic mechanisms were added to the previous list: sympathetic hyperactivity, cardiotonic steroids, nonosmotic sodium retention, and catalytic or labile iron. Also sympathetic hyperactivity within CKD caused progression of renal damage and also cardiovascular morbidity. Treatment of over activity of sympathetic nervous system could be an option to decrease deleterious effects of sympathetic nervous system in cases of chronic kidney disease ${ }^{(\mathbf{8})}$. 
In animal models having chronic kidney disease, cardiotonic steroids levels were increased above normal values, and led to hypertension development and could be a cause of uremic cardiomyopathy. Sodium storage within the skin, not accompanied by water, which is called non-osmotic sodium stores, have been proved to be a cause of hypertension occurrence, exacerbation of chronic kidney disorders, and development of cardiac and vascular system morbidity ${ }^{(\boldsymbol{9})}$.

LVH in cases of chronic kidney disease is accompanied by increased collagen deposition between myocardial fibrils (partially due to increased aldosterone level) and some sort of conversion to fibrous tissue. It could also be due to hyperparathyroidism, endothelin-1, catecholamines, and cardiotonic steroids. In addition to the previously mentioned histological changes, myocyte apoptosis/narcosis occurs leading to decrease in the number of working myocytes. This is accompanied by microvascular abnormalities including increased small arteriolar wall thickness and great decrease in the available capillary network supplying the heart (and this is specific to uremia) ${ }^{(\mathbf{1 0}, \mathbf{1 1})}$.

Sudden cardiac arrest and death could occur as a consequence of LVH and increased fibrous tissue deposition within cardiac tissue. Sudden death could be due to aberrant channels for electrical current pathway through the fibrous tissue deposited within the heart and this does occur in patient on regular hemodialysis sessions for three times weekly, and could happen to continuous ambulatory peritoneal dialysis (CAPD) patients. Heart muscle decreased blood supply disorders (ischemic disorders) come in second place after electrical current disturbance in causing sudden death in hemodialysis patients. Congestive heart failure occurring in chronic kidney disease states and dialyzed patients, is a main cause of mortality and it occurs in advanced stages of both LVH and heart muscle fibrosis (12).

\section{Vascular changes in chronic kidney disease:}

Blood vessels disorders are well known within chronic renal disease patients, since the data reported by Richard Bright in 1827, about uremic patients having increased aortic wall thickness. Arterial pulse wave velocity (PWV) is increased, meaning the presence of stiffness and reduced elasticity of large arteries in uremic patients. Increased arterial wall thickness and greater intima media thickness (IMT) than normal within chronic renal disease patients, calcium deposition within vascular walls, also within renal patients ${ }^{(\mathbf{1 3})}$.

Calcium deposition within blood vessel walls has got a causal relationship to the occurrence of cardiovascular events and death within chronic kidney disease patients. Vascular wall calcification is not a passive process but an active process, comprising the transformation of smooth muscle cell within arterial wall into a bone forming cell (like osteoblast) ${ }^{(\mathbf{1 4})}$.
Arterial wall rigidity, endothelial dysfunction, and calcium deposition within blood vessel walls, have got a relationship to fibroblast growth factor 23 (FGF23) levels in serum. Its value as a predictor of cardiac and vascular system disease, and phosphate-induced toxicity is not yet proved. When calcium salt precipitation occurs around atheromatous changes in the form of a plaque, within arteries intima layer, it is called atherosclerosis. When this occurs within the media layer of arteries, it is called arteriosclerosis or Monckeberg's sclerosis ${ }^{(15)}$.

Coronary vasculature pathology is a characteristic feature of chronic renal patients, and we need non-invasive methods to detect those patients within this category who are prone to cardiac events. Cardiac troponin T (cTnT) has been revealed as strong marker of cardiac vasculature pathology within hemodialysis patients, even those who are not complaining. The same authors reported that neutrophil gelatinase-associated lipocalin (NGAL), has been correlated to renal function loss and also cardiac and vascular system pathological changes within renal patients ${ }^{(16) .}$

\section{Clinical presentation of cardiovascular disease in chronic kidney disease:}

Herzog et al. (12), in their study, have mentioned that cardiac and vascular pathological states associated with chronic renal disease were as follows: coronary artery disease (CAD), myocardial infarction (MI), congestive heart failure (CHF), cerebrovascular disease (CVD), peripheral arterial disease (PAD), and sudden cardiac death (SCD).

\section{Coronary artery disease and myocardial infarction: Epidemiology and pathophysiology:}

Nakano et al. ${ }^{(17)}$, in their study showed a positive correlation existing between the degree of impairment of GFR and the degree of impaired cardiac blood supply. The presence of calcium deposition within coronary vasculature, multiple arterial affection was present. Randomized coronary angiographic studies performed on limited numbers of hemodialysis patients, have shown that more than $50 \%$ of patients enrolled within these studies had multiple coronary artery involvement.

Cardiac patients with renal impairment carry a potential for worse outcome than those without renal disease. Cardiac events and death have showed an inverse correlation with GFR less than $15 \mathrm{ml} / \mathrm{min}$ per $1.73 \mathrm{~m}^{2}$, as so much reduced GFR was found to be an independent predicting factor of morbidity and mortality within cardiac patients. The frequent occurrence of cardiac and vascular system disorders, together with cardiac death is usual with chronic renal patients, although the predisposing alterations occurring within body homeostasis in case of chronic renal disease, does not fully explain this situation. Atheromatous plaque occurrence and disruption have shown a relationship to inflammation and oxidative stress ${ }^{(18)}$. 


\section{Congestive heart failure: \\ Epidemiology:}

The following heart disorders are common to be found within chronic renal patients, as reported by Ronco et al. ${ }^{(19)}$ : first, congestive heart failure, followed by myocardial blood supply compromise, and then arrhythmias affecting heart conduction system (commonly atrial fibrillation). Death rates are much higher with diastolic than systolic dysfunction, occurring in the instance of congestive cardiac failure (19).

\section{Pathophysiology:}

Cardiomyopathy and cardiac failure occurrence within chronic renal disease patients, takes place through three known pathways: Cardiac afterload (also known as pressure overload), cardiac preload (also known as volume overload), together with biochemical and homeostatic changes affecting the myocardium in a milieu progressing towards uremia. Both higher than normal blood pressure level and also vessel wall rigidity usually lead to increased cardiac afterload, putting the heart under strain. Hemodynamic stress exercised over left cardiac compartment, mainly the ventricle and occurring due to preload and afterload, is accused of being the main cause of changes in cardiac muscle configuration and function. The biochemical and homeostatic changes occurring within chronic renal disease cause exacerbation of these changes ${ }^{(20)}$.

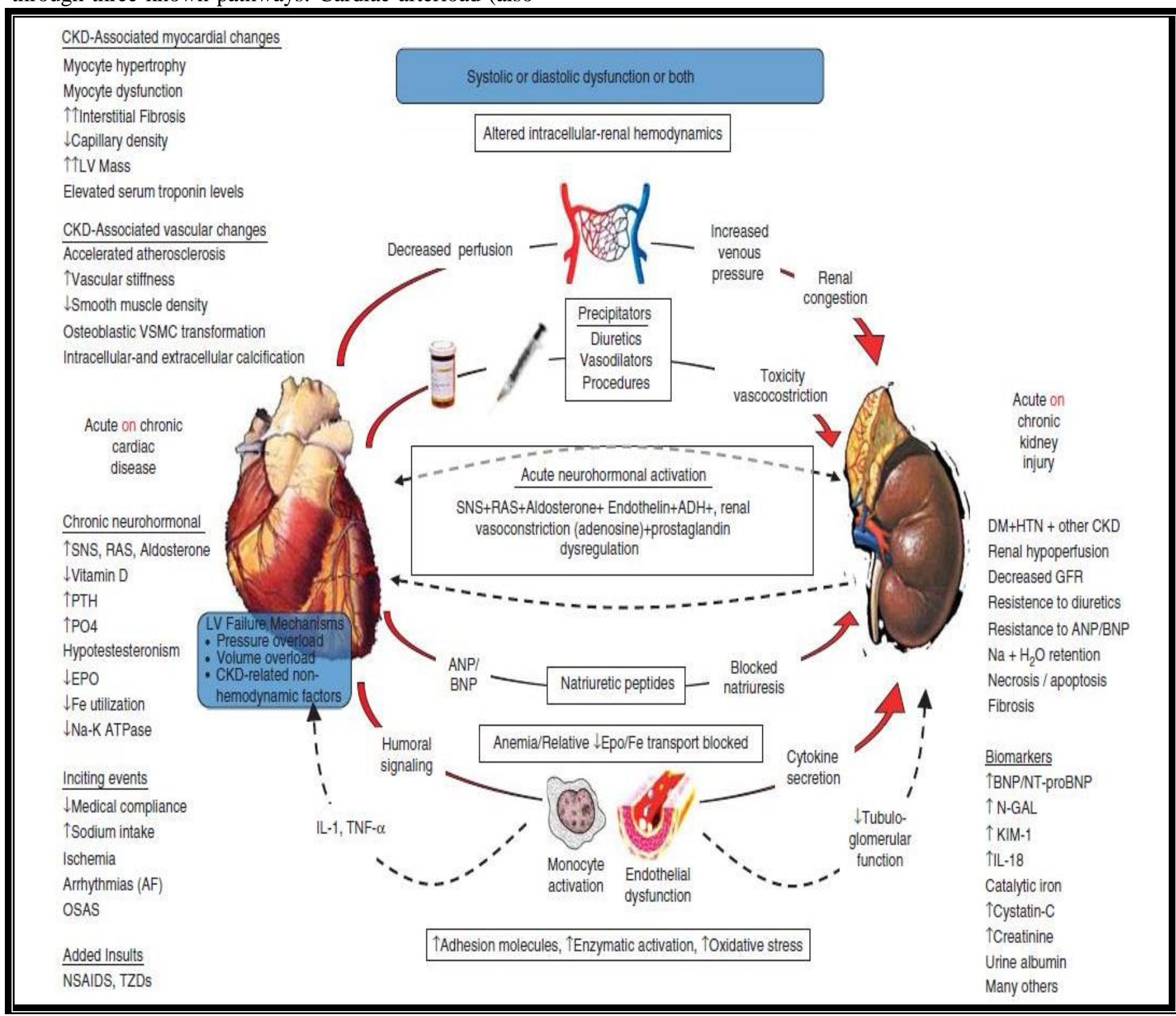

Figure (1): Cardio-Renal Syndrome Pathophysiology ${ }^{(21)}$. ADH, antidiuretic hormone; ANP, atrial natriuretic peptide; BNP, B-type natriuretic peptide; CKD, chronic kidney disease; DM, diabetes mellitus; EPO, erythropoietin; HTN, hypertension; IL-1, interleukin-1; KIM-1, kidney injury molecule-1; LV, left ventricular; N-GAL, neutrophil gelatinase-associated lipocalin; NSAID, non-steroidal anti-inflammatory drug; OSAS, obstructive sleep apnea syndrome; PTH, parathyroid hormone; SNS, sympathetic nervous system; TNF- $\alpha$, tumor necrosis factor- $\alpha$; TZD, thiazolidinediones; VSMC, vascular smooth muscle cell. 


\section{Diagnosis:}

Clinically, chronic renal patients suffering from effort intolerance on performing mild exercise, or also having chronic fatigue at rest, together with edema of different grades, may be diagnosed as having either heart failure or volume overload, and it is difficult to settle down a definite diagnosis. Echocardiography (including three dimensional echocardiography) is capable of diagnosing such cases. Echocardiography is mandatory in chronic renal patients complaining from left ventricular increased muscle mass and its associated possible cardiac ischemia, together with or without systolic and diastolic cardiac dysfunction ${ }^{(22)}$.

Iwanaga and Miyazaki ${ }^{(23)}$, have reported that biomarkers of myocardial cell damage (cTns $\mathrm{T}$ and cTns I), showed high levels within chronic renal patients suffering from congestive heart failure, without a clear explanation. Roberts et al. ${ }^{(24)}$, showed that cTns $\mathrm{T}$ and cTns I are considered as indicators of all-cause death occurrence in chronic renal disease stage $5 \mathrm{D}$.

Iwanaga and Miyazaki (23), reported that biomarkers revealing heart and kidney interrelationship are neutrophil gelatinase-associated lipocalin (NGAL), Kidney injury molecule 1 (KIM 1), together with interleukin 18 (IL 18). Also, among other biomarkers we have: Galectin-3, together with mid-regional proadrenomedullin. We also have markers known from the start to affect heart and kidney, they include catalytic iron and markers of oxidative stress.

All previous factors act separately from GFR level. GFR decrease has an impact level of descending order upon the following markers: Tumor necrosis factor $\alpha$, followed by hematocrit, followed by interleukin 6 , followed by cTnT, followed by $\mathrm{Kt} / \mathrm{V}$ (urea), followed by pre-albumin, followed by urea reduction ratio, followed by serum albumin, and at last C-reactive protein. All previous markers are thought to be due to effects of chronic renal disease such as biochemical and homeostatic changes due to chronic illness, changes due to chronic inflammatory state, together with malnutrition ${ }^{(\mathbf{1 2})}$.

\section{Prevention and treatment:}

Prevention relies on blood pressure and volume control and modification of usual risk factors for CHF and CKD progression. Treatment of CHF in CKD patients using the European Society of Cardiology Guidelines is reasonable (25). However, these therapeutic strategies are not based on strong evidence, as CKD patients are not adequately represented in randomized controlled trials in CHF. Dietary salt restriction should be a mainstay of clinical counseling. With regard to pharmacological treatment, several considerations are appropriate ${ }^{(\mathbf{1 2})}$.

CHF patients with kidney dysfunction often retain excessive salt and water and require more intensive diuretic treatment than do CHF patients with normal kidney function. Aldosterone antagonists should be used with caution as they may cause significant hyperkalemia. Despite strong evidence from randomized clinical trials that suggests that treatment with Angiotensin-converting enzyme inhibitors (ACEIs) or angiotensin II receptor blockers (ARBs) reduces cardiovascular morbidity and mortality in CHF, there is little equivalent evidence for CKD patients. Randomized clinical trials of CHF management with ACEIs, ARBs, new direct renin inhibitors, and mineralocorticoid receptor blockers (with or without potassium-binding resins) are required for CKD patients. Specialist supervision is recommended for patients with GFR levels below $~ 30$ $\mathrm{ml} / \mathrm{min}$ per $1.73 \mathrm{~m}^{2}$. One small randomized trial supports the combined use of ACEI+ARBs in the treatment of CHF in CKD 5D patients. Recent data strongly support the use of bisoprolol or carvedilol in CKD patients with CHF. Other potentially useful management strategies include correcting anemia and minimizing vascular calcification. Anemia correction, aiming for hemoglobin levels $>10 \mathrm{~g} / \mathrm{dl}$, has been shown to reduce LVH in CKD patients, but total correction does not further improve LV geometry or cardiovascular outcome. Erythropoiesis-stimulating agents and/or intravenous iron may improve exercise tolerance but are without survival benefit. Control of calcium and phosphate concentrations is instrumental in minimizing vessel calcification. Concern about calcification due to calcium-containing phosphate binders is increasing (26).

Therefore, the use of non-calcium-containing phosphate binders may be advantageous, although data are insufficient to establish the comparative superiority of these agents over calcium-containing phosphate binders for cardiovascular end points in CKD. Achieving adequate vitamin D status and avoiding excessively high or low parathyroid hormone concentrations (oversuppression) are reasonable treatment goals, although their efficacy has not been demonstrated. For CKD 5D patients with CHF, adequate ultrafiltration should be coupled with dietary sodium restriction and lower dialysate sodium concentrations; more frequent or longer dialysis sessions may be beneficial. High-flow fistulae or grafts may cause high cardiac output states ${ }^{(\mathbf{1 2})}$.

Evidence for management of acute $\mathrm{CHF}$ in CKD is limited. Inotropic therapy (dobutamine, milrinone, levosimendan) may be considered in patients with worsening renal function secondary to the fall in cardiac output; however, routine use of inotropes or other adrenergic-stimulating agents for acute decompensated CHF is not indicated in CKD patients. Ultrafiltration may be useful in refractory congestion ${ }^{(\mathbf{1 2})}$.

\section{CONCLUSION}

Cardiovascular disease is frequently underdiagnosed and undertreated in patients with 
chronic kidney disease. This group of patients should, therefore, be acknowledged as having high cardiovascular risk that needs particular medical attention at an individual level.

Financial support and sponsorship: Nil.

Conflict of interest: Nil.

\section{REFERENCES}

1. Chen J, Muntner P, Hamm L et al. (2004): The metabolic syndrome and chronic kidney disease in U.S. adults. Ann Intern Med., 140:167-71.

2.Herzog C, Littrell K, Arko C et al. (2007): Clinical characteristics of dialysis patients with acute myocardial infarction in the United States: a collaborative project of the United States Renal Data System and the National Registry of Myocardial Infarction. Circulation, 116:146572.

3. Fink H, Ishani A, Taylor B et al. (2012): Screening for, monitoring, and treatment of chronic kidney disease stages 1 to 3: a systematic review for the U.S. Preventive Services Task Force and for an American College of Physicians Clinical Practice Guideline. Ann Intern Med., 156:570-581.

4. Keith D, Nichols G, Gullion C et al. (2004): Longitudinal follow-up and outcomes among a population with chronic kidney disease in a large managed care organization. Arch Intern Med., 164: 659-63.

5. Ninomiya T, Perkovic V, De Galan B et al. (2009): Albuminuria and kidney function independently predict cardiovascular and renal outcomes in diabetes. Journal of the American Society of Nephrology, 20(8):1813-1821.

6. McIntyre N, Taal M (2008): How to measure proteinuria? Current Opinion in Nephrology and Hypertension, 17(6):600-603.

7. Kottgen A, Russell S, Loehr L et al. (2013): Reduced kidney function as a risk factor for incident heart failure: The Atherosclerosis Risk in Communities (ARIC) study. Journal of the American Society of Nephrology, 18(4):1307-1315.

8. Rump L, Amann K, Orth S et al. (2016): Sympathetic overactivity in renal disease: a window to understand progression and cardiovascular complications of uraemia? Nephrology Dialysis Transplantation, 15(11):1735-1738.

9. Kennedy D, Vetteth S, Periyasamy S et al. (2012): Central role for the cardiotonic steroid marinobufagenin in the pathogenesis of experimental uremic cardiomyopathy. Hypertension, 47(3):488-495.

10.Amann K (2008): Media calcification and intima calcification are distinct entities in chronic kidney disease. Clinical Journal of the American Society of Nephrology, 3(6):1599-1605.

11.Losi M, Memoli B, Contaldi C et al. (2010): Myocardial fibrosis and diastolic dysfunction in patients on chronic haemodialysis. Nephrology Dialysis Transplantation, 25(6):1950-1954.

12.Herzog C, Asinger R, Berger A et al. (2011): Cardiovascular disease in chronic kidney disease. A clinical update from Kidney Disease: Improving Global Outcomes (KDIGO). Kidney Int., 80(6):572-86.
13. McCullough P, Chinnaiyan K (2009): Annual progression of coronary calcification in trials of preventive therapies a systematic review. Archives of Internal Medicine, 169(22):2064-2070.

14. Mizobuchi M, Towler D, Slatopolsky E et al. (2009): Vascular calcification: the killer of patients with chronic kidney disease. Journal of the American Society of Nephrology, 20(7):1453-1464.

15. Larsson T (2010): The role of FGF-23 in CKD-MBD and cardiovascular disease: friend or foe? Nephrology Dialysis Transplantation, 25(5):1376-1381.

16.Hayashi T, Obi Y, Kimura T et al. (2014): Cardiac troponin $\mathrm{T}$ predicts occult coronary artery stenosis in patients with chronic kidney disease at the start of renal replacement therapy. Nephrology Dialysis Transplantation, 23(9):2936-2942.

17. Nakano T, Ninomiya T, Sumiyoshi S et al. (2015): Association of kidney function with coronary atherosclerosis and calcification in autopsy samples from Japanese elders: the Hisayama study. Am J Kidney Dis., 55:21-30.

18. Bhatt D (2008): Anti-inflammatory agents and antioxidants as a possible 'third great wave' in cardiovascular secondary prevention. Am J Cardiol., 101: 4-13.

19. Ronco C, McCullough P, Anker S et al. (2010): Cardiorenal syndromes: report from the consensus conference of the acute dialysis quality initiative. Eur Heart J., 31: 703711.

20. Redheuil A, Yu W, Wu C et al. (2015): Reduced ascending aortic strain and distensibility: earliest manifestations of vascular aging in humans. Hypertension, 55: 319-326.

21. McCullough P, Ronco C, Anker S et al. (2010): Cardiorenal syndromes: report from the consensus conference of the acute dialysis quality initiative. Eur Heart J., 31:70311.

22.Pecoits-Filho R, Barberato S (2012): Echocardiography in chronic kidney disease: diagnostic and prognostic implications. Nephron Clin Pract., 114: 242-247.

23. Iwanaga Y, Miyazaki S (2014). Heart failure, chronic kidney disease, and biomarkers an integrated viewpoint. Circ J., 74: 1274-1282.

24. Roberts M, Hare D, Macmillan N et al. (2009): Serial increased cardiac troponin $\mathrm{T}$ predicts mortality in asymptomatic patients treated with chronic haemodialysis. Ann Clin Biochem., 46: 291-295.

25.Dickstein K, Cohen-Solal A, Filippatos G et al. (2008): ESC Guidelines for the Diagnosis and Treatment of Acute and Chronic Heart Failure 2008: the Task Force for the Diagnosis and Treatment of Acute and Chronic Heart Failure 2008 of the European Society of Cardiology. Developed in collaboration with the Heart Failure Association of the ESC (HFA) and endorsed by the European Society of Intensive Care Medicine (ESICM). Eur Heart J., 29: 2388-2442.

26.Desai A, Nissenson A, Chertow G et al. (2009): The relationship between laboratory-based outcome measures and mortality in end-stage renal disease: a systematic review. Hemodial Int., 13: 347-359. 